\title{
Effectiveness of a Web-Based Intervention in Reducing Depression and Sickness Absence: Randomized Controlled Trial
}

Till Beiwinkel ${ }^{1}$, MA; Tabea Eißing ${ }^{1}$, MA (Sociology), MSc; Nils-Torge Telle ${ }^{1}$, PhD; Elisabeth Siegmund-Schultze ${ }^{2}$, MD; Wulf Rössler ${ }^{1,3,4,5}$, MSc, MD

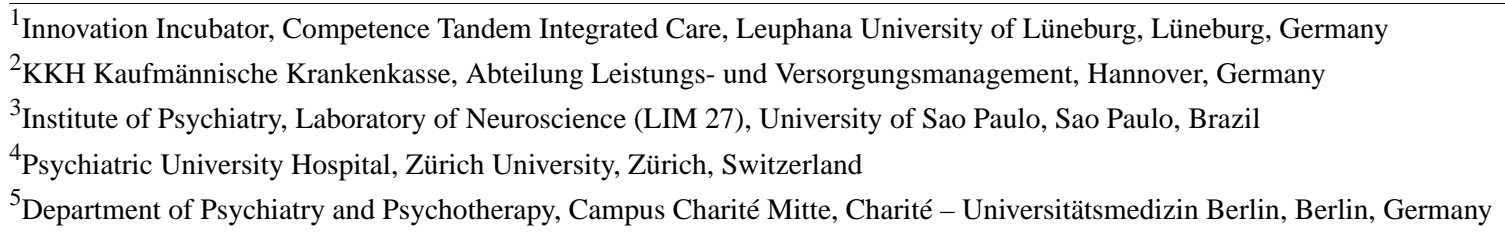

Corresponding Author:

Till Beiwinkel, MA

Innovation Incubator

Competence Tandem Integrated Care

Leuphana University of Lüneburg

Scharnhorststr 1

Lüneburg, 21335

Germany

Phone: 494131677 ext 7826

Fax: 4941316777601

Email: till.beiwinkel@leuphana.de

\section{Abstract}

Background: Depression is highly prevalent in the working population and is associated with significant loss of workdays; however, access to evidence-based treatment is limited.

Objective: This study evaluated the effectiveness of a Web-based intervention in reducing mild to moderate depression and sickness absence.

Methods: In an open-label randomized controlled trial, participants were recruited from a large-scale statutory health insurance and were assigned to two groups. The intervention group had access to a 12 week Web-based program consisting of structured interactive sessions and therapist support upon request. The wait-list control group had access to unguided Web-based psycho-education. Depressive symptoms were self-assessed at baseline, post-treatment, and follow-up (12 weeks after treatment) using the Patient Health Questionnaire (PHQ-9) and Beck Depression Inventory (BDI-II) as primary outcome measures. Data on sickness absence was retrieved from health insurance records. Intention-to-treat (ITT) analysis and per-protocol (PP) analysis were performed.

Results: Of the 180 participants who were randomized, 88 completed the post-assessment (retention rate: $48.8 \%, 88 / 180$ ). ITT analysis showed a significant between-group difference in depressive symptoms during post-treatment in favor of the intervention group, corresponding to a moderate effect size (PHQ-9: $d=0.55,95 \%$ CI $0.25-0.85, P<.001$, and BDI-II: $d=0.41$, CI 0.11-0.70, $P=.004)$. PP analysis partially supported this result, but showed a non-significant effect on one primary outcome (PHQ-9: $d=0.61$, $95 \%$ CI $0.15-1.07, P=.04$, and BDI-II: $d=0.2595 \% \mathrm{CI}-0.18$ to $0.65, P=.37$ ). Analysis of clinical significance using reliable change index revealed that significantly more participants who used the Web-based intervention $(63 \%, 63 / 100)$ responded to the treatment versus the control group $(33 \%, 27 / 80 ; P<.001)$. The number needed to treat (NNT) was 4.08 . Within both groups, there was a reduction in work absence frequency (IG: $-67.23 \%, P<.001, \mathrm{CG}:-82.61 \%, P<.001)$, but no statistical difference in sickness absence between groups was found $(P=.07)$.

Conclusions: The Web-based intervention was effective in reducing depressive symptoms among adults with sickness absence. As this trial achieved a lower power than calculated, its results should be replicated in a larger sample. Further validation of health insurance records as an outcome measure for eHealth trials is needed.

Trial Registration: International Standard Randomized Controlled Trial Number (ISRCTN): 02446836; http://www.isrctn.com/ISRCTN02446836 (Archived by WebCite at http://www.webcitation.org/6jx4SObnw) 
(J Med Internet Res 2017;19(6):e213) doi: 10.2196/jmir.6546

\section{KEYWORDS}

Internet; depression; absenteeism; cognitive therapy; randomized controlled trial

\section{Introduction}

Depression is highly prevalent in the working population [1]. It is estimated that over the course of one year, up to $26.7 \%$ of adults experience depressive symptoms and about $8.9 \%$ fulfill all criteria for a depressive disorder [2]. The resulting impairment and functional disability poses a substantial burden for the affected individual as well as the economy. Depressed employees have higher health care costs than those without depression [3,4], which in Europe contribute to a total estimated cost of 118 billion Euros per year [5].

Depression is linked to a high loss of work days [6]. In Germany, depression is a major driver of sickness absence and produces higher durations of sickness absence than other diagnoses of mental disorders [7]. When employees return to work after a depressive episode, distress often remains and performance is reduced [8,9]. Therefore, maintaining work capacity should be an important goal of clinical interventions. However, health promotion interventions targeting occupational health in employees with depression have been developed with mixed results [10-13]. Access to treatment remains limited, and the existing personal and structural barriers prevent those affected by depression from seeking timely, evidence-based help [14-16].

Web-based interventions are a promising tool to overcome the treatment gap in depression [17]. While generally using similar techniques as face-to-face therapy, such interventions are commonly delivered through websites and allow participants to access content at any time and work through lessons at their own pace. Web-based interventions vary in the level of therapist support [18], from entirely self-help to guided formats including regular therapist contact (eg, feedback via email). The advantages of Web-based interventions are their accessibility, a low threshold for help-seeking, relative anonymity, the patients' active role in (guided) self-help, and their low costs. However, in studies comparing Web-based interventions to usual care, risks associated with the dissemination of Web-based interventions have been reported as well $[19,20]$. Among the working population, Web-based interventions could especially benefit those who do not want to seek regular treatment because of negative perceptions of mental ill-health at the workplace.

The effectiveness of Web-based interventions in reducing depressive symptoms has been demonstrated repeatedly, but effect sizes vary considerably across studies [21-23]. For example, in the 19 studies that were included in the meta-analysis by Richards and Richardson [21], depression improvement in comparison with a control group ranged from no effect $(d=-0.03)$ to strong effects $(d=1.43)$. This heterogeneity makes it necessary to evaluate the interventions separately. Methodologically, weak control groups (eg, wait-list control instead of active control groups) and failure to employ intention-to-treat principles lead to an overestimation of the treatment effect [24]. Web-based interventions for depression have been studied among different clinical populations in Germany [25-29] but, to the best of our knowledge, no studies have yet focused on a Web-based intervention among a population with sick leave due to depression.

Participant self-reports are the primary outcome measure of eHealth trials. However, the lack of independent outcome assessments and the sole reliance on self-report measures limits this evolving field. For example, a report on the methodological quality of randomized controlled trials of Web-based interventions concluded that an increased use of independent outcome measurements is needed to improve the validity of efficacy studies [24]. To date, few studies employ independent outcomes and such attempts are limited to observer ratings of symptoms and do not extend to objective behavioral measurement of work absenteeism [30-32]. The lack of objective sickness absence measurements in research on Web-based interventions is surprising because sickness absence is frequently used as an integrated measure of health in other fields [33].

This study examined the effectiveness of a guided Web-based intervention in reducing depression and sickness absence among a high-risk population using both self-assessed depression and sickness absence assessments from health insurance records. We hypothesized that the Web-based intervention would be more effective in reducing depressive symptoms and sickness absence than the control group.

\section{Methods}

\section{Study Design}

This was a two-armed open-label randomized controlled trial. Participants were randomly assigned to either the intervention group (IG), with access to the guided Web-based intervention, or the wait-list control group (CG), with access to unguided Web-based psycho-education.

We used a computerized block randomization procedure (allocation ratio 1:1, block size 10). The researcher conducting the randomization had no information about the participants apart from their 6-digit codes and did not participate in the enrollment and assignment of the participants to study groups, which was handled by two different researchers. Outcome variables were assessed at baseline (T0) and 12 weeks after randomization (post-treatment, T1). In addition, a follow-up measurement was assessed 24 weeks after randomization (12 weeks after treatment, T2). Sample size calculation was based on expected between-group differences at follow-up. G*Power was used for sample size calculation [34]. First, we assumed a power of 0.80, an alpha level of 0.05 , and a small to medium effect size $(d=0.3)$, which results in $\mathrm{N}=357$ to perform a two-sided $t$ test for differences between two independent means. Second, adding $20 \%$ attrition rate at inclusion, post-assessment, and follow-up, we calculated that $\mathrm{N}=608$ participants needed to be enrolled. 
The study was approved by the ethical review board at Leuphana University of Lüneburg. The study was registered retrospectively on February 1, 2013, under the International Standard Randomized Controlled Trial Number ISRCTN02446836; http://www.controlled-trials.com/ISRCTN02446836. Despite retrospective registration, no participants were enrolled before registration.

\section{Recruitment}

Participants were recruited from a large-scale German statutory health insurance between January 2013 and April 2014, with the first participant enrolled in February 2013. We recruited members from Kaufmännische Krankenkasse (KKH), a statutory health insurance company with about 1.8 million members nationwide. First, to identify participants who were at high risk for sick leave due to depression, insurance members were screened for previous diagnosis of depression (International Classification of Disease codes F32.0, F32.1, F33.0, F33.1, and F34.1), previous sickness absence due to depression, and current sickness absence. Second, the study team sent an invitation letter to all positively screened insurance members along with study information, the informed consent form (see Multimedia Appendix 1), and a 6-digit code to login into the platform. Adults with a previous episode of mild to moderate depression (International Classification of Disease codes F32.0, F32.1, F33.0, F33.1) or dysthymia (F34.1) were included to avoid giving less intensive treatment than necessary. Before registration on the platform, a screening for exclusion criteria was performed. Participants with a score of $\geq 20$ on the Patient Health Questionnaire (PHQ-9), indicating severe depression, were excluded. A second exclusion criterion was suicidality as measured by one item on the presence of suicidal thoughts. All participants had unrestricted access to treatment as usual during the study period, including access to the treatments and services which are typically available for depression in the German health care system (eg, psychotherapy and medication).

\section{Intervention}

The Web-based intervention "HelpID" is a 12-week, Web-based program based on cognitive-behavioral therapy, awareness training, and systemic counseling. The program was structured into 12 weekly sessions. Each lasted 30 to 45 minutes and included interactive elements, videos, and audios that explained depression-related themes (eg, symptoms and course) as well as graphs, illustrations, exercises, and guidance for awareness and relaxation. Each session was available one week after completing the prior session. Participants received weekly reminder emails when a new session was available. The program had a guided format with therapist contact upon request, that is, psychologists (bachelor level or higher) trained in the intervention approach provided feedback via email or telephone. The intervention was developed by a team of clinical psychologists headed by Dr Despina Lion, a clinical psychologist and therapist with extensive experience in systemic counseling, cognitive-behavioral therapy, and neurological psychology. It is accessible online [35] (see Multimedia Appendix 2). Since July 2016, the copyright of "HelpID" is owned by IVPNetworks $\mathrm{GmbH}$, a private integrated care company. The intervention is commercially available to single users and is included in health care plans of statutory health insurances.

The intervention's psychological approach includes cognitive-behavioral therapy, mindfulness training, and systemic counseling. During the development process, current research evidence on the respective therapies was used as the basis, and special emphasis was placed on a "person-based" approach, focusing on the perspectives of the people who would use the intervention. Cognitive-behavioral therapy is the most extensively researched psychological treatment approach in Web-based interventions [36]. From cognitive-behavioral therapy, the intervention used elements of cognitive restructuring, with an emphasis on dealing with negative moods and automatic thoughts, as well as exercises for behavioral activation. Mindfulness training has been used increasingly in psychotherapy over the past years. It was shown to be effective for depressive symptoms and can be adapted to online formats $[37,38]$. The intervention module on mindfulness engages the user in exercises to observe the self and to practice mindfulness in daily situations. Systemic counseling is a therapeutic approach that highlights the social context surrounding the individual and its resources [39]. Specifically, systemic questioning techniques and instructions were employed to make use of the participants' social support. Systemic principles were presented in specific weekly sessions, while homework exercises on systemic therapy encouraged the participants to adopt a systemic viewpoint and behavior change in their everyday interactions.

\section{Control Group}

The control group was a wait-list plus psycho-education condition. During the 12-week study period, participants had access to text-based information on the nature of depression and its symptoms and treatment. The psycho-education content was developed by a team of trained psychologists (bachelor degree or higher) and was based upon scientific literature on depression (eg, the German S3-Guideline) [40]. This type of control condition was chosen because more active control groups (ie, psycho-education) are considered to be more methodologically valid than passive control groups (ie, wait-list conditions) [24]. There is evidence that psycho-education can reduce depressive symptoms and serve as an initial treatment in primary care [41]. The control group did not have access to therapist guidance. Participants were eligible to access the intervention after study completion, if they requested access.

\section{Outcomes}

The primary outcomes were self-assessed depressive symptoms with the Patient Health Questionnaire (PHQ-9) and the Beck Depression Inventory (BDI II). The PHQ-9 measured the severity of depressive symptoms over the preceding 2 weeks, resulting in a score between 0 to 27 points with higher values indicating more severe depression [42,43]. The PHQ-9 was shown to have good reliability and construct validity [42]. The BDI II uses 21 items to measure depression severity [44,45]. The BDI II showed good psychometric properties in German-speaking samples in regard to internal consistency, retest-reliability, and construct validity [46]. As a secondary outcome, quality of life was assessed using the Manchester Short Assessment of Quality of Life (MANSA) [47]—a 12-item 
scale rating the participants' satisfaction with different life domains. The MANSA has been validated in a Swedish sample and showed satisfactory internal consistency and construct validity [48]. User satisfaction was measured at post-assessment using the item "Overall, how satisfied are you with the program?" with four answer options: 1=very good, 2=good, $3=$ satisfactory, and $4=$ poor.

Information on work absenteeism was retrieved from health insurance records. In the German health care system, such standardized health data is collected routinely. Its primary purpose is cost reimbursement and quality assurance, but it can be made available for secondary analysis. Due to the routine data collection, health insurance records are assumed to have high ecological validity. We matched health insurance records from $\mathrm{KKH}$ health insurance with participants' data using a 6-digit participant code as identifier. The code was generated for each positively screened insurance member and was also used for registration on the study platform. We analyzed sickness absence data that covered the 90 days before randomization (baseline) and 90 days after intervention (post-assessment). Three sickness absence measures were constructed according to Hensing et al [33]. First, the number of persons who were absent at least once, second, absence frequency as the number of times a person was absent during the 90 day period irrelevant of duration, and third, absence duration as the total number of absence days during the 90 day period. Sickness absence data was not diagnosis-specific.

\section{Statistical Analysis}

Statistical analysis was performed on an intention-to-treat (ITT) basis according to the recommendations in the CONSORT statement [49] and its adaption for eHealth trials [50] (see Multimedia Appendix 3). Missing data at post-treatment was imputed using the Markov Chain Monte Carlo multiple imputation (missing data module in IBM SPSS version 22), where 10 estimations per missing value were specified and, besides the outcome variables, group assignment was included as an additional variable. Under the assumption that data is missing at random, multiple imputation was considered suitable to produce more precise estimates of the true intervention effect than other imputation methods, that is, last observation carried forward [51]. In addition, per-protocol (PP) analysis was performed to examine the robustness and sensitivity of the findings when including only participants who completed the post-assessment. $t$ tests were used to determine differences in baseline characteristics and for within-group differences. The difference in the intervention outcomes between the intervention group and the control group at post-treatment was estimated using analysis of covariance (ANCOVA) with baseline scores as the covariate. Cohen $d$ was calculated as a measure of the effect size, using pooled standard deviations [52]. For the between-group effect sizes, Cohen $d$ was computed from the mean differences.

To assess clinical significance on an individual level, the reliable change index (RCI) was computed for the PHQ-9 [53]. Cronbach alpha $=.89$ from Kroenke et al [42] was used as an estimate of the reliability of the PHQ-9, along with pre-treatment standard deviations from the current study. Participants were classified as "responders" if they displayed a reliable positive change, or as "deteriorated" if they displayed a negative change on the RCI. A reliable positive change corresponds to less than -1.96 on the RCI and a change of PHQ-9 points to greater than -4.05 . A reliable negative change corresponds to less than -1.96 on the RCI and a change in PHQ-9 points to greater than 4.05. Finally, the number needed to treat (NNT) [54] was computed.

All analysis was performed using Stata 13 . The reported $P$ values are two sided and in the $95 \%$ CI. 
Figure 1. Study flowchart.

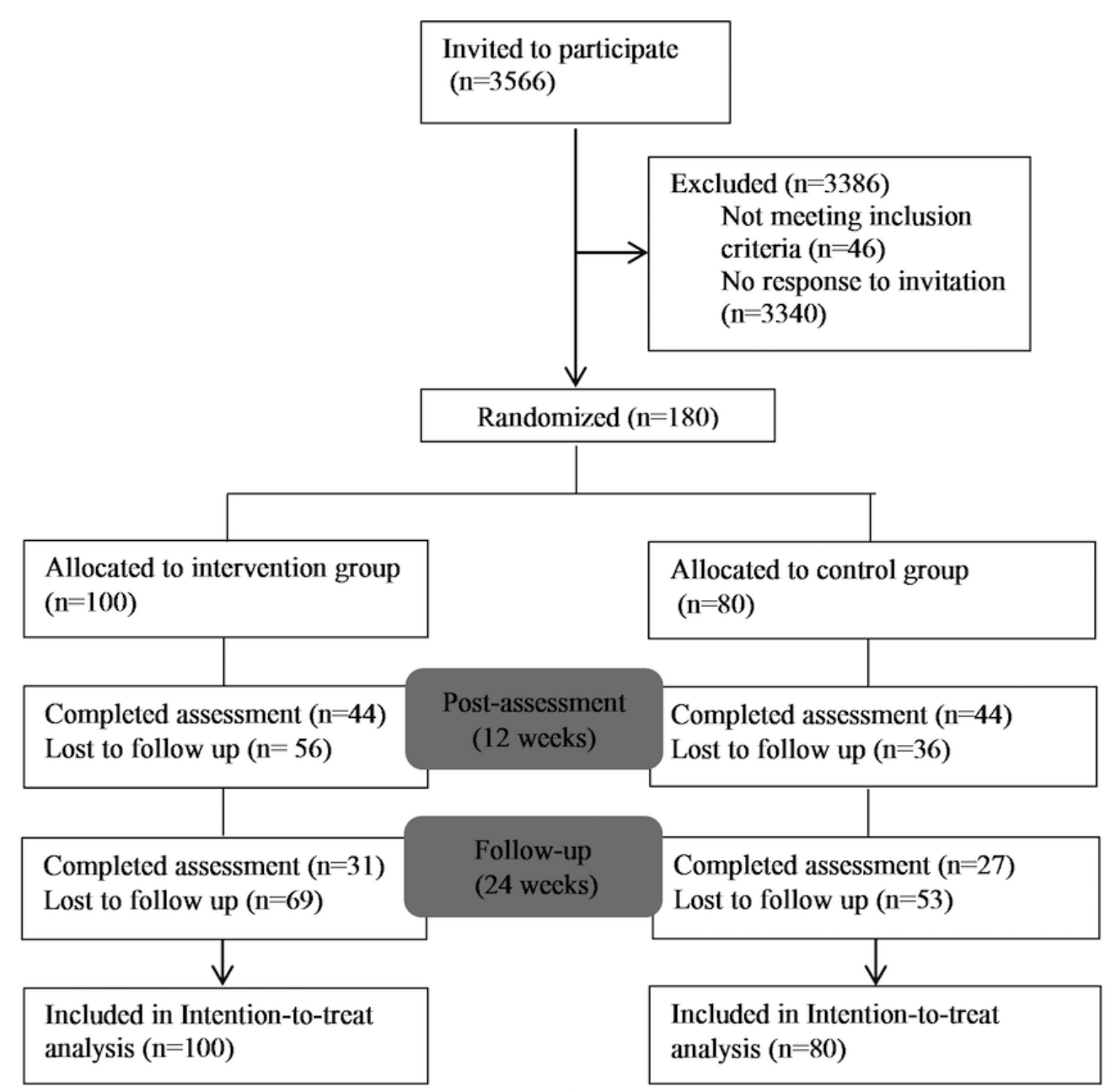

\section{Results}

\section{Participant Characteristics}

Figure 1 shows the flow of participants through the study. The complete pool of insurance members was screened, which resulted in 3929 positively screened insurance members who were subsequently invited to participate. Of those, 180 responded, met the inclusion criteria, provided informed consent, and were randomized. Of the 180 participants, 88 completed the post-assessment after 12 weeks (retention rate: $48.8 \%$, $88 / 180$ ), and 58 completed the follow-up assessment after 24 weeks (retention rate: $32.2 \%, 58 / 180$ ). To estimate achieved power, a post-hoc power analysis was conducted. This revealed that with the sample of 180 participants, the achieved power to detect an effect of $d=0.3$ was 0.51 .

Baseline characteristics between participants who completed the post-assessments and those who were lost to follow-up were tested for differences. Older participants (PHQ at T1: $P=.02$, BDI at T2: $P=.03$ ) and participants with higher education (PHQ at T1: $P=.03$, BDI at T2: $P=.04$ ) were more likely to complete the post-assessment on the primary outcome and the follow-up assessment. Participants who were not in psychotherapy during study enrollment were more likely to complete post-assessment on one of the primary outcomes (BDI at T1: $P=.04$ ) and the follow-up assessment (BDI at T2: $P=.04$ ) as compared with participants who were on a waiting list or in psychotherapy at enrollment. No other relevant differences between those who completed the post-assessments and those who were lost to follow-up were found.

Table 1 shows the participant characteristics at baseline. Participants had an average age of 48 years and were predominantly female $(68 \%)$. The majority were married or had a partner $(56 \%)$ and had completed secondary education or higher $(85 \%)$. About half of the sample was working full-time $(51 \%)$ and another $27 \%$ were working part time, while $20 \%$ of those working had an executive position. About one-fifth (21\%) were not working. The majority had experienced a previous depressive episode $(51 \%)$ or reported to have chronic depression (30\%). During study enrollment, half of the participants were prescribed with depression medication (50\%), 26\% were in psychotherapy, and $13 \%$ were on a psychotherapy waiting list. Of the total number of enrolled participants, $43 \%$ had a sick leave certificate at the time. The mean baseline depressive symptoms in the sample were 11.10 points on the PHQ-9 $(\mathrm{SD}=4.45)$, indicating moderate depression. No clinically relevant differences in terms of any baseline characteristics were found, and we concluded that randomization was successful. 
Table 1. Participant characteristics at baseline.

\begin{tabular}{|c|c|c|c|c|}
\hline Characteristic & $\begin{array}{l}\text { Intervention group } \\
\mathrm{n}=100^{\mathrm{a}}\end{array}$ & $\begin{array}{l}\text { Control group } \\
n=80\end{array}$ & $\begin{array}{l}\text { Total sample } \\
\mathrm{n}=180\end{array}$ & $P$ value \\
\hline PHQ-9 Score, mean (SD) & $11.53(4.35)$ & $10.56(4.53)$ & $11.10(4.45)$ & .15 \\
\hline Age, mean (SD) & $47.01(10.36)$ & $48.66(11.59)$ & $47.74(10.92)$ & .31 \\
\hline \multicolumn{5}{|l|}{ Gender, n (\%) } \\
\hline Male & $34(34.0)$ & $23(28.7)$ & $57(31.7)$ & .45 \\
\hline Female & $66(66.0)$ & $57(71.3)$ & $123(68.3)$ & \\
\hline \multicolumn{5}{|l|}{ Relationship, n (\%) } \\
\hline Single & $24(24.0)$ & $18(22.5)$ & $42(23.3)$ & .93 \\
\hline Married/Partner & $56(56.0)$ & $46(57.5)$ & $102(56.7)$ & \\
\hline Divorced/Separated & $15(15.0)$ & $14(17.5)$ & $29(16.1)$ & \\
\hline Widowed & $5(5.0)$ & $2(2.5)$ & $7(3.9)$ & \\
\hline \multicolumn{5}{|l|}{ Education, $\mathbf{n}(\%)$} \\
\hline Low & $13(13.3)$ & $13(16.2)$ & $26(14.6)$ & .38 \\
\hline Middle & $63(64.3)$ & $42(52.5)$ & $105(59.0)$ & \\
\hline High & $22(22.4)$ & $25(31.3)$ & $47(26.4)$ & \\
\hline \multicolumn{5}{|l|}{ Employment, n (\%) } \\
\hline Full-time & $51(52.0)$ & $39(50.6)$ & $90(51.4)$ & .41 \\
\hline Part-time & $30(30.6)$ & $18(23.4)$ & $48(27.4)$ & \\
\hline Not working & $17(17.3)$ & $20(26.0)$ & $37(21.2)$ & \\
\hline \multicolumn{5}{|l|}{ Executive position, $\mathrm{n}(\%)$} \\
\hline Yes & $20(22.0)$ & $13(17.6)$ & $33(20.0)$ & .48 \\
\hline No & $71(78.0)$ & $61(82.4)$ & $132(80.0)$ & \\
\hline \multicolumn{5}{|c|}{ Previous depression $^{b}, \mathbf{n}(\%)$} \\
\hline None & $18(18.0)$ & $14(17.7)$ & $32(17.9)$ & .53 \\
\hline Episodic & $54(54.0)$ & $38(48.1)$ & $92(51.4)$ & \\
\hline Chronic & $28(28.0)$ & $27(34.2)$ & $55(30.7)$ & \\
\hline \multicolumn{5}{|c|}{ Depression medication, $\mathrm{n}(\%)$} \\
\hline Yes & $53(53.0)$ & $36(45.6)$ & $89(49.7)$ & .33 \\
\hline No & $47(47.0)$ & $43(54.4)$ & $90(50.3)$ & \\
\hline \multicolumn{5}{|l|}{ In psychotherapy, n (\%) } \\
\hline Yes & $30(30.0)$ & $16(20.3)$ & $46(25.7)$ & .15 \\
\hline No & $57(57.0)$ & $52(65.8)$ & $109(60.9)$ & \\
\hline Waiting List & $13(13.0)$ & $11(13.9)$ & $24(13.4)$ & \\
\hline
\end{tabular}

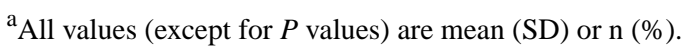

bOriginal item: "Did you have these symptoms for the first time?" Answer options: 1. "Yes," 2. "No; I had one or multiple episodes," 3. "No; the symptoms last for several years."

\section{Intervention Effectiveness}

Table 2 shows the mean scores, standard deviations for the intervention outcomes at baseline and at post-assessment, effect sizes, and statistical significance, based on the intention-to-treat sample (imputed data). A significant between-group difference in favor of the intervention group was found for the PHQ-9 $\left(F_{1,179}=15.06, P<.001\right)$, which corresponds to a medium effect size $(d=0.55$, CI $0.25-0.85)$. For those in BDI-II, a significant between-group difference at post-treatment was found $\left(F_{1,179}=8.69, P=.004\right)$, which corresponds to a moderate effect size $(d=0.40$, CI $0.10-0.70)$. 
Table 2. Means, standard deviations (SD), effect sizes, and statistical significance for intervention outcomes based on intention-to-treat sample (imputed data).

\begin{tabular}{|c|c|c|c|c|c|c|}
\hline \multirow[t]{2}{*}{ Outcome } & \multicolumn{3}{|l|}{ Mean (SD) } & \multirow{2}{*}{$\begin{array}{l}\text { Effect Size }^{\mathrm{a}} \\
\text { Cohen } d(95 \% \mathrm{CI})\end{array}$} & \multicolumn{2}{|c|}{ Statistical Significance ${ }^{b}$} \\
\hline & Baseline (T0) & Post-assessment (T1) & Difference (T0-T1) & & $F$ Value & $P$ value \\
\hline \multicolumn{7}{|l|}{ PHQ-9 } \\
\hline Intervention & $11.53(4.35)$ & $6.51(2.87)$ & $-5.02(3.62)$ & $0.55(0.25-0.85)$ & 15.06 & $<.001$ \\
\hline Control & $10.56(4.53)$ & $7.76(3.63)$ & $-2.80(4.42)$ & & & \\
\hline \multicolumn{7}{|l|}{ BDI-II } \\
\hline Intervention & $20.07(7.99)$ & $13.55(6.46)$ & $-6.17(6.39)$ & $0.41(0.11-0.70)$ & 8.69 & .004 \\
\hline Control & $18.78(9.84)$ & $15.52(8.62)$ & $-3.56(6.68)$ & & & \\
\hline \multicolumn{7}{|l|}{ MANSA } \\
\hline Intervention & $3.27(0.72)$ & $3.50(0.67)$ & $0.14(0.71)$ & 0,12 & 0.72 & .39 \\
\hline Control & $3.30(0.85)$ & $3.44(0.70)$ & $0.22(0.55)$ & $(-0.17$ to 0.42$)$ & & \\
\hline
\end{tabular}

${ }^{\mathrm{a}}$ Between-group effect size from mean differences.

${ }^{b}$ Based on ANCOVA controlling for baseline scores (T0).

In addition, both the intervention and the control group showed reductions in depressive symptoms as measured by within-group changes from baseline to post-assessment. In the intervention group, a mean reduction of 5 points on the PHQ-9 was found $\left(t_{99}=14.28, P<.001\right)$, which corresponds to a large within-group effect size $(d=1.42$, CI 1.14-1.71). In the control group, a mean reduction of 2.79 points was found $\left(t_{79}=5.82, P<.001\right)$, corresponding to a moderate effect size $(d=0.65$, CI $0.41-.89)$.

In the per-protocol analysis, we tested for differences in PHQ-9 scores between intervention completers and noncompleters at post-assessment. No significant difference was found $\left(t_{178}=-.28\right.$, $P=$.78). Table 3 presents the results of the per-protocol analysis.

For the PHQ-9, a significant between-group difference in favor of the intervention group was found among completers (PHQ-9: $\left.F_{1,77}=8.98, P=.04\right)$, corresponding to a moderate effect size $(d=0.61$, CI $0.15-1.07)$. The mean PHQ-9 scores among completers were reduced by 5.70 points in the intervention group and by 2.24 points in the control group - this corresponds to a large effect size $(d=1.72$, CI 1.23-2.22) and a moderate effect size ( $d=0.49$, CI $0.14-0.82)$ for within-group changes, respectively. For the BDI-II, the between-group effect failed to reach statistical significance in the per-protocol analysis $\left(F_{1,77}=0.81, \quad P=.37, d=0.25, \quad\right.$ CI -0.18 to 0.65$)$. BDI-II within-group changes were significant in the intervention group $\left(t_{43}=3.68, P<.001\right)$ and the control group $\left(t_{42}=4.70, P<.001\right)$.

Table 3. Means, standard deviations (SD), effect sizes, and statistical significance for intervention outcomes based on per-protocol sample (nonimputed data).

\begin{tabular}{|c|c|c|c|c|c|c|}
\hline \multirow[t]{2}{*}{ Outcome } & \multicolumn{3}{|l|}{ Mean (SD) } & \multirow{2}{*}{$\begin{array}{l}\text { Effect Size }^{\mathrm{a}} \\
\text { Cohen } d(95 \% \mathrm{CI})\end{array}$} & \multicolumn{2}{|c|}{ Statistical Significance $^{b}$} \\
\hline & Baseline (T0) & Post-assessment (T1) & Difference (T0-T1) & & $F$ value & $P$ value \\
\hline \multicolumn{7}{|l|}{ PHQ-9 } \\
\hline Intervention & $11.53(4.35)$ & $6.50(3.85)$ & $-5.03(3.29)$ & \multirow{2}{*}{$0.61(0.15-1.07)$} & \multirow[t]{2}{*}{8.98} & \multirow[t]{2}{*}{.04} \\
\hline Control & $10.56(4.53)$ & $7.95(4.62)$ & $-2.61(4.61)$ & & & \\
\hline \multicolumn{7}{|l|}{ BDI-II } \\
\hline Intervention & $20.07(7.99)$ & $14.86(8.05)$ & $-5.21(7.59)$ & \multirow{2}{*}{$\begin{array}{l}0.25 \\
(-0.18 \text { to } 0.65)\end{array}$} & \multirow[t]{2}{*}{0.81} & \multirow[t]{2}{*}{.37} \\
\hline Control & $18.78(9.84)$ & $15.32(10.34)$ & $-3.46(6.32)$ & & & \\
\hline \multicolumn{7}{|l|}{ MANSA } \\
\hline Intervention & $3.27(0.72)$ & $3.52(0.86)$ & $0.25(0.64)$ & \multirow{2}{*}{$\begin{array}{l}0.18 \\
(-0.22 \text { to } 0.58)\end{array}$} & \multirow[t]{2}{*}{0.34} & \multirow[t]{2}{*}{.56} \\
\hline Control & $3.30(0.85)$ & $3.42(0.83)$ & $0.12(0.79)$ & & & \\
\hline
\end{tabular}

${ }^{\mathrm{a}}$ Between-group effect size from mean differences.

${ }^{\mathrm{b}}$ Based on ANCOVA controlling for baseline scores (T0). 
Table 4. Work absence indicators at baseline and at post-assessment for 160 participants.

\begin{tabular}{|c|c|c|c|c|}
\hline Indicator & Baseline (T0) $^{\mathrm{a}}$ & Post-assessment (T1) & Difference $(\%)$ & $P$ value \\
\hline \multicolumn{5}{|c|}{ Absence at least once, n (\%) } \\
\hline Intervention & $75(85.22)$ & $25(28.41)$ & $-50(-66.67)$ & $<.001$ \\
\hline Control & $58(80.55)$ & $12(16.67)$ & $-46(-79.31)$ & $<.001$ \\
\hline \multicolumn{5}{|c|}{ Absence frequency, mean (SD) } \\
\hline Intervention & $1.19(0.09)$ & $0.39(0.08)$ & $-0.80(-67.23)$ & $<.001$ \\
\hline Control & $1.15(0.10)$ & $0.20(0.06)$ & $-0.95(-82.61)$ & $<.001$ \\
\hline \multicolumn{5}{|c|}{ Absence duration, mean (SD) } \\
\hline Intervention & $25.60(2.03)$ & $24.65(3.80)$ & $-0.95(-3.71)$ & .79 \\
\hline Control & $27.69(2.37)$ & $24.04(4.36)$ & $-3.65(-13.18)$ & .34 \\
\hline
\end{tabular}

${ }^{\mathrm{a}}$ Baseline and post-assessment cover a period of 90 days each.

\section{Treatment Response}

In the intervention group, $63 \%(63 / 100)$ of the participants showed a reliable symptom change from baseline to post-intervention and were thus classified as responders. In the control group, $33 \%$ (27/80) were classified as responders. The difference in reliable symptom change between intervention and control group was significant $\left(t_{178}=3.39, P<.001\right)$. This resulted in a NNT of 4.08. One participant in the intervention group experienced symptom deterioration, and five participants in the control group experienced symptom deterioration.

\section{Sickness Absence}

Information on sickness absenteeism was available for 160 participants (Intervention group: $n=88$, control group: $n=72$ ). For 20 participants, sickness absenteeism could not be retrieved from insurance records, and therefore the data from these participants was unavailable.

Table 4 shows persons who were absent at least once, with absence frequency and absence duration at baseline and at post-assessment. The majority of the participants were absent at least once during the baseline period: IV: $85 \%$ (77/85), CG: $80 \%$ (58/72). Overall, significantly fewer participants were absent at least once during post-assessment (IV: $28 \%, \mathrm{CG}$ : $16 \%)$. The within-group absence reductions were significant (IV: $\left.t_{87}=6.54, P<.001, \mathrm{CG}: t_{71}=6.17, P<.001\right)$.

Regarding absence frequency, participants in the intervention group were absent on average 1.2 times at baseline and 0.4 times at post-assessment. In the control group, participants were absent 1.2 times at baseline and 0.2 times at post-assessment. The within-group reductions in absence frequency were significant (IV: $\left.t_{87}=7.49, P<.001, \mathrm{CG}: t_{71}=8.59, P<.001\right)$.

Similarly, high absence durations at both baseline and post-assessment were found. From the 90 days examined at each time point, participants in the intervention group were absent from work 26 days at baseline and 25 days at post-assessment. In the control group, participants were absent 28 days at baseline and 24 days at post-assessment. However, there were no significant differences in absence duration from baseline to post-assessment (IV: $t_{87}=.26, P=.79, \mathrm{CG}: t_{71}=.95$,
$P=.34$ ). For all three measurements, the between-group differences at post-assessment failed to reach statistical significance (absence at least once: $F_{1,159}=.80, P=.37$, absence frequency: $F_{1,159}=3.24, P=.07$, absence duration: $F_{1,159}=.02$, $P=$.88).

\section{Secondary Outcome}

No significant difference in quality of life as measured by MANSA was found $\left(F_{1,169}=.71, P=.40, d=0.13, \mathrm{CI}-0.21\right.$ to $0.41)$.

\section{Long-term effect}

No significant between-group difference for BDI-II depression scores at 24-week follow-up was found $\left(F_{1,85}=.81, P=.33\right)$. However, significant within-group changes were sustained for both the intervention and the control group. In the intervention group, there was a mean reduction from baseline to follow-up assessment of 5.46 points on the BDI-II $\left(t_{99}=6.81, P<.001\right)$, which corresponds to a moderate effect size $(d=0.68, \mathrm{CI}$ 0.46-0.90). In the control group, there was a mean reduction of 4.69 points $\left(t_{79}=4.37, P<.001\right)$, corresponding to a small effect size $(d=0.48$, CI $0.26-0.72)$.

\section{User Satisfaction}

87 participants completed the user satisfaction survey at post-assessment. In the intervention group, $13.6 \%(6 / 44)$ rated the program overall as very good, $68.2 \%(30 / 44)$ as good, and $18.2 \%(8 / 44)$ as satisfactory. In the control group, $4.6 \%(2 / 43)$ rated the program as very good, $37.2 \%(16 / 43)$ as good, $34.9 \%$ as satisfactory, and $23.3 \%(10 / 43)$ as poor. Mean satisfaction scores were 2.04 in the intervention group and 2.76 in control group. There was a significantly higher mean satisfaction in the intervention group $\left(t_{85}=4.60, P<.001\right)$.

\section{Discussion}

\section{Principal Findings}

This study compared the effectiveness of a Web-based intervention in reducing depressive symptoms and sickness absence among adults with immediate risk for sickness absence due to mild to moderate depression. When compared with a 
wait-list plus psycho-education control group, participants who used the Web-based intervention showed a significantly greater reduction in depressive symptoms. However, because of the low response and high attrition in this study, one primary outcome did not reach statistical significance among participants who completed the intervention (per-protocol analysis) and at follow-up, only within-group changes were sustained but the intervention effect was not. In terms of individual clinical significance, significantly more participants in the intervention group responded to the treatment. We used health insurance records to measure sickness absence and found that sickness absenteeism declined in both groups, but there were no statistical differences in work absence between groups. The achieved power of this trial was lower than calculated. Therefore, its results should be replicated in a larger sample.

\section{Comparison With Prior Work}

These findings are especially relevant when considering the increasing impact of mental ill-health on workforces across industrialized countries [55]. Depression is a significant cause for workday losses and produces more absence durations than other mental illnesses. To reduce the illness burden, widespread access to evidence-based treatment is needed to maintain workers' mental health before companies and individuals face more serious burdens as the illness progresses. In general, Web-based interventions provide a promising treatment tool because these interventions can be accessed at any time and at different locations at the users' own pace. Due to their relative anonymity, Web-based interventions may especially benefit employees with depression who wish to avoid the negative perceptions of being mentally ill in the workplace. However, risks associated with the dissemination of Web-based interventions in the health care system have been reported as well. According to meta-analysis, the effects of Web-based interventions vary, making it necessary to evaluate each intervention separately. If Web-based interventions that are effective in reducing depressive symptoms are more widely implemented and adopted, a positive impact on the burden and impairment caused by depression can be expected. It can also help to overcome the shortcomings of conventional treatment (eg, waiting lists).

This study contributes to the growing body of research that supports the effectiveness of Web-based interventions for depression. Within this research, a critical mass of efficacy studies is needed to identify subgroups for which these interventions work [56]. Adults who are at high risk for sick leave from work due to mild to moderate depression have not yet been targeted specifically by Web-based interventions. In terms of effect sizes, previous research has found significant heterogeneity between studies. This makes it necessary to evaluate each intervention separately. The effect sizes reported in this study are comparable to other studies evaluating guided interventions for depression, including those included in the meta-analysis by Andersson and Cuijpers [23] where a mean between-group effect size of $d=0.41$ is reported. The amount of therapy guidance that is necessary to increase intervention effectiveness and adherence remains a subject of debate in the field of Web-based interventions [18,57]. Considering that this intervention provided only minimal therapist support upon request and achieved similar outcomes to studies with more intense guidance, we speculate that merely having the option to contact a therapist during the intervention-versus regular therapist contact-is sufficient for the needs of many participants and works equally well. However, it is possible that the number of dropouts could have been reduced with regular guidance. The examination of support preferences was not within the scope of this study and further research on this subject is needed.

We found that health insurance records are a suitable outcome for effectiveness research in Web-based interventions. Both groups showed reductions in work absence frequency, however, no statistical difference in work absence between groups was found. Several explanations may account for this finding. First, we cannot rule out the possibility that the decline in work absence frequency over time was caused by regression toward the mean or spontaneous remission. Our sample was recruited during a period of high levels of sickness absence, as seen in the data (see Table 3). Consequently, due to statistical chance, the frequency of sickness absence tended to approach lower levels at post-assessment. A healthy control group is needed to compare baseline levels of sickness absence, which was unavailable in this study. Second, it is possible that the 90-day time period in our study was not sufficiently long to appropriately detect changes in work absence. Previous population studies on sickness absence due to mental health problems found a median absence duration of 79 days [58]. This indicates that sickness absence started or ended outside of the time period of this study. Similarly, our sample was not adequately powered to detect small differences in work loss days. Third, organizational factors (ie, high work demands, job security) could have influenced work absence in this study. Unfortunately, we could not measure organizational variables.

To disentangle these explanations, future studies on the effect of Web-based interventions on work absence should include a longer time period, information on organizational factors that may be related to sickness absence, and work absence data from a healthy control group for baseline comparisons. Integrating objective behavioral parameters (ie, sickness absence data from health insurance companies) can increase the validity of effectiveness studies and might be a valuable addition to self-reported outcome measurements.

\section{Privacy and Data Security}

In Web-based interventions, health-related information is processed and stored electronically. Therefore, data security and confidentiality issues need to be taken seriously. This study used several measures to ensure the privacy of the study participants. Person-related information and study data were stored on separate servers to ensure that individuals could not be identified. Communication between the users' Web browsers and the servers were encrypted via a Secure Sockets Layer (SSL) connection. All data were stored on servers located in Germany.

\section{Limitations}

There are several limitations to this study that must be acknowledged. First, although reporting the effect of the Web-based intervention was within the scope of our study, it 
remains unclear as to which specific elements and properties of the intervention contributed to its effectiveness. Regarding the length of the intervention, evidence on the dose-response relationship in psychotherapy points to the conclusion that most progress occurs in the first few sessions of an intervention [59]. Similarly, Web-based interventions with 8 or less sessions were found to be more effective than interventions with more than 8 sessions [21]. This indicates that the present intervention with its 12 sessions could be shortened in length while maintaining its effectiveness.

A second limitation concerns the response rate. Response from the pool of positively screened insurance members was low $(5.8 \%, 226 / 3929)$. This raises the concern that participants were particularly motivated to use a Web-based intervention when compared with nonparticipants, especially because nonparticipants were positively screened for sickness absence due to depression and thus belonged to the target group. Ideally, data from nonparticipants should have been collected as a baseline comparison group, but this data was unavailable in this study because of a lack of informed consent. When the complete pool of insurance members was screened and all positively screened insurance members were invited, recruitment was stopped. This resulted in a substantially smaller sample than was previously calculated in the power analysis (calculated $\mathrm{N}=680$ vs actual $\mathrm{N}=180$ ).

Third, attrition during the study was high. At post-assessment, $45.5 \%$ of the participants had dropped out, and at follow-up, $67.7 \%$ of participants had dropped out. In general, dropout is a common problem in Web-based interventions [60]. However, the dropout in this study was remarkably higher than the average attrition rate for Web-based interventions with therapist support, as reported in the meta-analysis by Richards and Richardson (28\%) [21]. Several recent studies on Web-based interventions showed dropout rates that were remarkably lower [27,32]. One possibility for the relatively high attrition in this study is that participants who failed to complete a weekly session were reminded via email only. Comparable studies, which used telephone reminders, achieved substantially higher participant compliance. A second explanation is that therapist guidance was available upon request only. Guided interventions have lower attrition rates as compared to unguided interventions. Thus, it is possible that participants who were at risk for dropout were less likely to use therapist guidance. Despite our analysis of per protocol and imputed data showing comparable results, which indicates no difference in intervention completers versus noncompleters, the risk remains that study dropout could have biased the results. Due to the associations of several baseline characteristics (age, education, and being in psychotherapy) with the likelihood to complete the outcome assessments, the missing at random assumption could have been violated. Overall, the high attrition rates limit the conclusions drawn from this study.

Third, the positive relationship of age and education with study dropout seen here limits the generalizability of the findings to younger and less educated groups. This is supported by the composition of the study sample, where highly educated participants and those in executive positions are over-represented. Further studies with more statistical power are needed to identify effectiveness among different subgroups.

Fourth, participants had access to treatment as usual during the study, including psychotherapy and medication. Therefore we cannot exclude the possibility that within-group changes in depression scores were affected by third factor variables. Thus, within-group changes must be interpreted with caution.

Fifth, no clinical interviews were conducted to assess depression. Structured clinical interviews represent the gold standard of clinical assessment, with superior validity and reliability. Due to limited resources, this study relied solely on participants' self-reports to assess clinical symptoms.

Sixth, the amount and duration of provided therapeutic support during the study was not measured, making it difficult to compare the results with other studies on Web-based interventions that have used different levels of support, ranging from no support to more intensive and regular support. In this study, support was provided upon request, which could have prevented some participants from using support, thus lowering adherence.

Seventh, we used a wait-list plus psycho-education control group. Wait-list control groups undermine internal validity and may lead to an over-estimation of the treatment effect [24]. Thus, active control groups are considered to be less biased. To maximize participant response, we decided to inform control group participants that they could access the intervention upon request after study completion. This may have lowered expectations with regard to the control condition. Furthermore, control group participants were active during the study period as they had access to psycho-education. As a result, we observed mean symptom reductions in the control group, which is consistent with the finding that Web-based psycho-education can reduce depressive symptoms [41]. At the same time, it is possible that psycho-education has adverse effects in some participants, because it sensitizes patients to the topic of depression, leading to an over-reporting of symptom severity at follow-up. For example, a study that used a psycho-education control group found that the incidence of depression was higher than usual [32]. Our results show that reliable symptom deterioration was low overall, but occurred more frequently in the control group $(6.25 \%, 5 / 80)$ as compared with the intervention group $(1 \%, 1 / 100)$. This suggests that adverse effects in the control groups were present, but were unlikely to bias the treatment effect. As we did not collect data on usage and engagement with the psycho-education in the control condition, the perceived credibility of the psycho-education remains unknown.

Eighth, this was an open-label trial, where participants and researchers were aware of which group was receiving which treatment. Furthermore, only questionnaire data was assessed as a proxy of use parameters, but no uptake data was available on the actual usage of the program (eg, frequency and length of website usage). Data on how the participants interacted with the program could provide valuable insights into the effectiveness of specific intervention elements. 


\section{Conclusions}

The Web-based intervention reduced depressive symptoms among adults with sickness absence. As this trial achieved a lower power than calculated, its results should be replicated in a larger sample. Further validation of health insurance records as an outcome measure for eHealth trials is needed.

\section{Acknowledgments}

This research was funded by the European Union Innovation Incubator within the European Regional Development Fund. Novego AG is the developer and commercial distributor of the Web-based intervention, "HelpID." Since July 2016, the copyright of "HelpID" has been owned by IVPNetworks GmbH. In cooperation with the Innovation Incubator, the developer made the intervention available at no cost to the participants in the trial. Funder, developer, and copyright owner had no role in the study design, data analysis, decision to publish, or preparation of the manuscript. KKH health insurance provided access to its insurance members for the purposes of this trial. $\mathrm{KKH}$, the authors, and the funder have no financial interests in the distribution of the intervention. The authors wish to thank the entire team that worked on the study, including Jörn Moock, Sally Kindermann, Kai Kossow, Andreas Maier, Claudia Schmidt, Neele Garbers, Sharlina Spiering, Mathias von Waldenfels, Despina Lion, and Manuela Stockmann.

\section{Conflicts of Interest}

This research was funded by the European Union Innovation Incubator, and one goal of the Innovation Incubator was to facilitate regional economic development by evaluating the intervention, HelpID, produced by Novego AG, a regional commercial partner within the Innovation Incubator.

\section{Multimedia Appendix 1}

Patient information informed consent form.

[PDF File (Adobe PDF File), 113KB-Multimedia Appendix 1]

\section{Multimedia Appendix 2}

Intervention screenshots.

[ZIP File (Zip Archive), 631KB-Multimedia Appendix 2]

\section{Multimedia Appendix 3}

CONSORT-EHEALTH-checklist.

\section{[PDF File (Adobe PDF File), 806KB-Multimedia Appendix 3]}

\section{References}

1. Sanderson K, Andrews G. Common mental disorders in the workforce: recent findings from descriptive and social epidemiology. Can J Psychiatry 2006 Feb;51(2):63-75. [doi: 10.1177/070674370605100202]

2. Wittchen H, Jacobi F. Epidemiologie der Depression. In: Stoppe G, editor. Volkskrankheit Depression?: Bestandsaufnahme und Perspektiven (German Edition). Heidelberg: Springer; 2006.

3. Goetzel RZ, Anderson DR, Whitmer RW, Ozminkowski RJ, Dunn RL, Wasserman J, Health Enhancement Research Organization (HERO) Research Committee. The relationship between modifiable health risks and health care expenditures. An analysis of the multi-employer HERO health risk and cost database. J Occup Environ Med 1998 Oct;40(10):843-854. [Medline: 9800168]

4. Welch CA, Czerwinski D, Ghimire B, Bertsimas D. Depression and costs of health care. Psychosomatics 2009;50(4):392-401. [doi: 10.1176/appi.psy.50.4.392] [Medline: 19687180 ]

5. Sobocki P, Jönsson B, Angst J, Rehnberg C. Cost of depression in Europe. J Ment Health Policy Econ 2006 Jun;9(2):87-98. [Medline: 17007486]

6. Kessler RC, Frank RG. The impact of psychiatric disorders on work loss days. Psychol Med 1997 Jul;27(4):861-873. [Medline: 9234464]

7. Kliner K, Rennert D, Richter M, editors. BKK Gesundheitsatlas 2015: Gesundheit in Regionen - Blickpunkt Psyche. Berlin: MWV Medizinisch Wissenschaftliche Verlagsgesellschaft und BKK Dachverband e.V; 2015.

8. Stewart WF, Ricci JA, Chee E, Hahn SR, Morganstein D. Cost of lost productive work time among US workers with depression. JAMA 2003 Jun 18;289(23):3135-3144. [doi: 10.1001/jama.289.23.3135] [Medline: 12813119]

9. Wang PS, Beck AL, Berglund P, McKenas DK, Pronk NP, Simon GE, et al. Effects of major depression on moment-in-time work performance. Am J Psychiatry 2004 Oct;161(10):1885-1891. [doi: 10.1176/ajp.161.10.1885] [Medline: 15465987] 
10. Blonk RW, Brenninkmeijer V, Lagerveld SE, Houtman ILD. Return to work: a comparison of two cognitive behavioural interventions in cases of work-related psychological complaints among the self-employed. Work \& Stress 2006 Apr;20(2):129-144. [doi: 10.1080/02678370600856615]

11. Nieuwenhuijsen K, Bültmann U, Neumeyer-Gromen A, Verhoeven AC, Verbeek JH, van der Feltz-Cornelis CM. Interventions to improve occupational health in depressed people. Cochrane Database Syst Rev 2008(2):CD006237. [doi: 10.1002/14651858.CD006237.pub2] [Medline: 18425942]

12. Martin A, Sanderson K, Cocker F. Meta-analysis of the effects of health promotion intervention in the workplace on depression and anxiety symptoms. Scand J Work Environ Health 2009 Jan;35(1):7-18 [FREE Full text] [Medline: 19065280]

13. Kawohl W, Moock J, Heuchert S, Rössler W. Job maintenance by supported employment: an overview of the "supported employment plus" trial. Front Public Health 2015;3:140 [FREE Full text] [doi: 10.3389/fpubh.2015.00140] [Medline: 26075194]

14. Glozier N. Workplace effects of the stigmatization of depression. J Occup Environ Med 1998 Sep;40(9):793-800. [Medline: 9777563]

15. Greenberg PE, Kessler RC, Birnbaum HG, Leong SA, Lowe SW, Berglund PA, et al. The economic burden of depression in the United States: how did it change between 1990 and 2000? J Clin Psychiatry 2003 Dec;64(12):1465-1475. [Medline: 14728109]

16. Mohr DC, Ho J, Duffecy J, Baron KG, Lehman KA, Jin L, et al. Perceived barriers to psychological treatments and their relationship to depression. J Clin Psychol 2010 Apr;66(4):394-409 [FREE Full text] [doi: 10.1002/jclp.20659] [Medline: 20127795]

17. Riper H, Andersson G, Christensen H, Cuijpers P, Lange A, Eysenbach G. Theme issue on e-mental health: a growing field in internet research. J Med Internet Res 2010;12(5):e74 [FREE Full text] [doi: 10.2196/jmir.1713] [Medline: 21169177]

18. Newman MG, Szkodny LE, Llera SJ, Przeworski A. A review of technology-assisted self-help and minimal contact therapies for anxiety and depression: is human contact necessary for therapeutic efficacy? Clin Psychol Rev 2011 Feb;31(1):89-103. [doi: 10.1016/j.cpr.2010.09.008] [Medline: 21130939]

19. Gilbody S, Littlewood E, Hewitt C, Brierley G, Tharmanathan P, Araya R, et al. Computerised cognitive behaviour therapy $(\mathrm{cCBT})$ as treatment for depression in primary care (REEACT trial): large scale pragmatic randomised controlled trial. BMJ 2015 Nov 11;351:1-13. [doi: 10.1136/bmj.h5627]

20. Kenter R, van de Ven PM, Cuijpers P, Koole G, Niamat S, Gerrits R. Costs and effects of Internet cognitive behavioral treatment blended with face-to-face treatment: results from a naturalistic study. Internet Interv 2015;2(1):77-83. [doi: 10.1016/j.invent.2015.01.001]

21. Richards D, Richardson T. Computer-based psychological treatments for depression: a systematic review and meta-analysis. Clin Psychol Rev 2012 Jun;32(4):329-342. [doi: 10.1016/j.cpr.2012.02.004] [Medline: 22466510]

22. Van't HE, Cuijpers P, Stein DJ. Self-help and internet-guided interventions in depression and anxiety disorders: a systematic review of meta-analyses. CNS Spectr 2009 Feb;14(2 Suppl 3):34-40. [Medline: 19238128]

23. Andersson G, Cuijpers P. Internet-based and other computerized psychological treatments for adult depression: a meta-analysis. Cogn Behav Ther 2009;38(4):196-205. [doi: 10.1080/16506070903318960] [Medline: 20183695]

24. Kiluk BD, Sugarman DE, Nich C, Gibbons CJ, Martino S, Rounsaville BJ, et al. A methodological analysis of randomized clinical trials of computer-assisted therapies for psychiatric disorders: toward improved standards for an emerging field. Am J Psychiatry 2011 Aug;168(8):790-799 [FREE Full text] [doi: 10.1176/appi.ajp.2011.10101443] [Medline: 21536689]

25. Meyer B, Berger T, Caspar F, Beevers CG, Andersson G, Weiss M. Effectiveness of a novel integrative online treatment for depression (Deprexis): randomized controlled trial. J Med Internet Res 2009;11(2):e15 [FREE Full text] [doi: 10.2196/jmir.1151] [Medline: 19632969]

26. Buntrock C, Ebert D, Lehr D, Riper H, Smit F, Cuijpers P, et al. Effectiveness of a web-based cognitive behavioural intervention for subthreshold depression: pragmatic randomised controlled trial. Psychother Psychosom 2015;84(6):348-358. [doi: 10.1159/000438673] [Medline: 26398885]

27. Nobis S, Lehr D, Ebert DD, Baumeister H, Snoek F, Riper H, et al. Efficacy of a web-based intervention with mobile phone support in treating depressive symptoms in adults with type 1 and type 2 diabetes: a randomized controlled trial. Diabetes Care 2015 May;38(5):776-783. [doi: 10.2337/dc14-1728] [Medline: 25710923]

28. Ebert DD, Lehr D, Baumeister H, Boß L, Riper H, Cuijpers P, et al. GET.ON Mood Enhancer: efficacy of Internet-based guided self-help compared to psychoeducation for depression: an investigator-blinded randomised controlled trial. Trials 2014;15:39 [FREE Full text] [doi: 10.1186/1745-6215-15-39] [Medline: 24476555]

29. Klein JP, Berger T, Schröder J, Späth C, Meyer B, Caspar F, et al. Effects of a psychological internet intervention in the treatment of mild to moderate depressive symptoms: results of the EVIDENT study, a randomized controlled trial. Psychother Psychosom 2016;85(4):218-228. [doi: 10.1159/000445355] [Medline: 27230863]

30. Thiart H, Lehr D, Ebert DD, Berking M, Riper H. Log in and breathe out: internet-based recovery training for sleepless employees with work-related strain - results of a randomized controlled trial. Scand J Work Environ Health 2015 Mar;41(2):164-174. [doi: 10.5271/sjweh.3478] [Medline: 25590336] 
31. Geraedts AS, Kleiboer AM, Twisk J, Wiezer NM, van MW, Cuijpers P. Long-term results of a web-based guided self-help intervention for employees with depressive symptoms: randomized controlled trial. J Med Internet Res 2014;16(7):e168 [FREE Full text] [doi: 10.2196/jmir.3539] [Medline: 25008127]

32. Buntrock C, Ebert DD, Lehr D, Smit F, Riper H, Berking M, et al. Effect of a web-based guided self-help intervention for prevention of major depression in adults with subthreshold depression: a randomized clinical trial. JAMA 2016 May 3;315(17):1854-1863. [doi: 10.1001/jama.2016.4326] [Medline: 27139058]

33. Hensing G, Alexanderson K, Allebeck P, Bjurulf P. How to measure sickness absence? literature review and suggestion of five basic measures. Scand J Soc Med 1998 Jun;26(2):133-144. [Medline: 9658514]

34. Faul F, Erdfelder E, Lang A, Buchner A. G*Power 3: a flexible statistical power analysis program for the social, behavioral, and biomedical sciences. Behav Res Methods 2007 May;39(2):175-191. [doi: 10.3758/BF03193146]

35. von Waldenfels M, Muth D, Pudack T. Novego. 2011. Online-unterstützungsprogramme von NovegoWochen-programm helpID URL: https://www.novego.de/programme/ [accessed 2016-08-22] [WebCite Cache ID 6jwzh2xt1]

36. Cuijpers P, van Straten A, Andersson G. Internet-administered cognitive behavior therapy for health problems: a systematic review. J Behav Med 2008;31(2):169-177. [doi: 10.1007/s10865-007-9144-1]

37. Kuyken W, Warren F, Taylor R, Whalley B, Crane C, Bondolfi G. Efficacy of mindfulness-based cognitive therapy in prevention of depressive relapse: an individual patient data meta-analysis from randomized trials. JAMA psychiatry 2016;73(6):565-574. [doi: 10.1001/jamapsychiatry.2016.0076]

38. Kvillemo P, Brandberg Y, Bränström R. Feasibility and outcomes of an internet-based mindfulness training program: a pilot randomized controlled trial. JMIR Ment Health 2016 Jul 22;3(3):e33 [FREE Full text] [doi: 10.2196/mental.5457] [Medline: 27450466]

39. Schweitzer J, Von Schlippe A. Lehrbuch der systemischen Therapie und Beratung. Göttingen: Vandenhoeck \& Ruprecht; 2007.

40. Leitlinien. 2015 Jun. S3-Leitlinie/Nationale VersorgungsLeitlinie: Unipolare Depression - Langfassung URL: http://www. leitlinien.de/mdb/downloads/nvl/depression/archiv/depression-1aufl-vers5-lang.pdf [accessed 2017-06-09] [WebCite Cache ID 6r5M8L6p8]

41. Donker T, Griffiths KM, Cuijpers P, Christensen H. Psychoeducation for depression, anxiety and psychological distress: a meta-analysis. BMC Med 2009;7:79 [FREE Full text] [doi: 10.1186/1741-7015-7-79] [Medline: 20015347]

42. Kroenke K, Spitzer RL, Williams JB. The PHQ-9: validity of a brief depression severity measure. J Gen Intern Med 2001 Sep;16(9):606-613 [FREE Full text] [Medline: 11556941]

43. Löwe B, Spitzer R, Zipfel S, Herzog W. Gesundheitsfragebogen für Patienten (PHQ-D). Manual und Testunterlagen. Karlsruhe: Pfizer; 2002.

44. Beck A, Steer R, Brown G. Manual for the beck depression inventory-II. San Antonio, TX: Psychological Corporation; 1996.

45. Hautzinger M, Keller F, Kühner C. Beck Depressions Inventar II (BDI 2). Revision. Frankfurt: Harcourt Test Services; 2006.

46. Kühner C, Bürger C, Keller F, Hautzinger M. [Reliability and validity of the revised beck depression inventory (BDI-II). results from German samples]. Nervenarzt 2007 Jun;78(6):651-656. [doi: 10.1007/s00115-006-2098-7] [Medline: 16832698]

47. Priebe S, Huxley P, Knight S, Evans S. Application and results of the manchester short assessment of quality of life (Mansa). Int J Soc Psychiatry 1999 Mar 01;45(1):7-12. [doi: 10.1177/002076409904500102]

48. Björkman T, Svensson B. Quality of life in people with severe mental illness. Reliability and validity of the Manchester short assessment of quality of life (MANSA). Nord J Psychiatry 2005 Jul;59(4):302-306. [doi: 10.1080/08039480500213733] [Medline: $\underline{16195135]}$

49. Moher D, Hopewell S, Schulz K, Montori V, Gotzsche P, Devereaux P, et al. CONSORT 2010 explanation and elaboration: updated guidelines for reporting parallel group randomised trials. BMJ 2010 Mar 23;340:c869. [doi: 10.1136/bmj.c869] [Medline: 20332511]

50. Eysenbach G, CONSORT-EHEALTH Group. CONSORT-EHEALTH: improving and standardizing evaluation reports of Web-based and mobile health interventions. J Med Internet Res 2011;13(4):e126 [FREE Full text] [doi: 10.2196/jmir.1923] [Medline: 22209829]

51. Schafer JL, Graham JW. Missing data: our view of the state of the art. Psychol Methods 2002 Jun;7(2):147-177. [Medline: 12090408 ]

52. Cohen J. Statistical power analysis for the behavioral sciences. Hillsdale, NJ: L. Erlbaum Associates; 1988.

53. Jacobson NS, Truax P. Clinical significance: a statistical approach to defining meaningful change in psychotherapy research. J Consult Clin Psychol 1991 Feb;59(1):12-19. [Medline: 2002127]

54. Cook RJ, Sackett DL. The number needed to treat: a clinically useful measure of treatment effect. BMJ 1995 Feb 18;310(6977):452-454 [FREE Full text] [Medline: 7873954$]$

55. OECD. Sick on the job?: myths and realities about mental health and work. Paris, France: OECD Publishing; 2012.

56. Lehr D, Geraedts A, Asplund R, Khadjesari Z, Heber E, Bloom J. Occupational e-mental health: current approaches promising perspectives for promoting mental health in workers. In: Wiencke M, Cacace M, Fischer S, editors. Healthy at Work. Interdisciplinary Perspectives. Switzerland: Springer International Publishing; 2016. 
57. Zarski A, Lehr D, Berking M, Riper H, Cuijpers P, Ebert DD. Adherence to internet-based mobile-supported stress management: a pooled analysis of individual participant data from three randomized controlled trials. J Med Internet Res 2016;18(6):e146 [FREE Full text] [doi: 10.2196/jmir.4493] [Medline: 27357528]

58. Nystuen P, Hagen KB, Herrin J. Mental health problems as a cause of long-term sick leave in the Norwegian workforce. Scand J Public Health 2001 Sep;29(3):175-182. [Medline: 11680768]

59. Stiles WB, Barkham M, Connell J, Mellor-Clark J. Responsive regulation of treatment duration in routine practice in United Kingdom primary care settings: replication in a larger sample. J Consult Clin Psychol 2008 Apr;76(2):298-305. [doi: 10.1037/0022-006X.76.2.298] [Medline: 18377125]

60. Eysenbach G. The law of attrition. J Med Internet Res 2005;7(1):e11 [FREE Full text] [doi: 10.2196/jmir.7.1.e11] [Medline: $\underline{15829473]}$

\author{
Abbreviations \\ ANCOVA: analysis of covariance \\ BDI-II: Beck Depression Inventory \\ ITT: intention-to-treat \\ KKH: Kaufmännische Krankenkasse \\ MANSA: Manchester Short Assessment of Quality of Life \\ PHQ-9: Patient Health Questionnaire \\ PP: per-protocol \\ RCI: Reliable Change Index
}

\author{
Edited by G Eysenbach; submitted 25.08.16; peer-reviewed by T Krieger, B Meyer, C Buntrock; comments to author 05.12.16; revised \\ version received 20.01.17; accepted 27.03.17; published 15.06.17 \\ Please cite as: \\ Beiwinkel T, Eißing T, Telle NT, Siegmund-Schultze E, Rössler W \\ Effectiveness of a Web-Based Intervention in Reducing Depression and Sickness Absence: Randomized Controlled Trial \\ J Med Internet Res 2017;19(6):e213 \\ URL: http://www.jmir.org/2017/6/e213/ \\ doi: 10.2196/jmir.6546 \\ PMID: 28619701
}

(CTill Beiwinkel, Tabea Eißing, Nils-Torge Telle, Elisabeth Siegmund-Schultze, Wulf Rössler. Originally published in the Journal of Medical Internet Research (http://www.jmir.org), 15.06.2017. This is an open-access article distributed under the terms of the Creative Commons Attribution License (https://creativecommons.org/licenses/by/4.0/), which permits unrestricted use, distribution, and reproduction in any medium, provided the original work, first published in the Journal of Medical Internet Research, is properly cited. The complete bibliographic information, a link to the original publication on http://www.jmir.org/, as well as this copyright and license information must be included. 